\title{
FROBENIUS MANIFOLDS FROM YANG-MILLS INSTANTONS
}

\author{
JAN SEGERT
}

\begin{abstract}
We present an elementary self-contained account of semisimple Frobenius manifolds in three dimensions, and exhibit a new family of explicit examples. These examples are constructed from Yang-Mills instantons with a certain symmetry.
\end{abstract}

\section{Introduction}

The concept of a Frobenius manifold was introduced and extensively developed by Dubrovin, whose lecture notes [D1] constitute the primary reference for Frobenius manifolds and many of the applications. The lecture notes of Hitchin [Hi1] and of Manin [Mn1] are also very good references, as are the recent papers of Manin [Mn3] and of Dubrovin [D2]. Frobenius manifolds have appeared in a remarkably wide range of settings, including quantum cohomology [RuT], mirror symmetry and variation of Hodge structure $[G]$, unfoldings of singularities $[\mathrm{Au}, \mathrm{Sa}]$, and the WDVV equation of topological quantum field theory [D1]. Since Frobenius manifolds are relevant in the description of some deep geometrical phenomena, it is not surprising that explicit solutions of the Frobenius manifold equations are rather difficult to construct.

In this paper we present new Frobenius manifolds of dimension three. The paper is self-contained, no previous knowledge of Frobenius manifolds is assumed. In Section 2 we define semisimple Frobenius manifolds in the framework of canonical coordinates. In Section 3 we exhibit explicit formulae for the new Frobenius manifolds. In Section 4 and the Appendix we use elementary Riemannian geometry to prove some of the fundamental results for Frobenius manifolds [D1] for the special case of dimension three. The cross-product effects numerous simplifications that are specific to dimension three. Using these results, Proposition 2.2 in particular, it is easy to verify that the explicit formulae presented in Section 3 are indeed Frobenius manifolds.

The geometry used to construct the new Frobenius manifolds will be discussed in detail in another publication. Here we give only a brief outline. The theory of isomonodromic deformations offers an approach to the construction of semisimple Frobenius manifolds [D1,Hi,Mn1,Sa]. Isomonodromic deformations

Received January 15, 1998.

Research partially supported by NSF Grant DMS-9404468 
of a meromorphic connection on $\mathbb{C} P^{1}$ are well-understood [Ml], but explicit examples are difficult to construct. This difficulty is reflected in the correspondence between solutions of the Painlevé VI differential equation and a class of isomonodromic deformation $[\mathrm{F}, \mathrm{JM}]$; Painlevé equations are notoriously difficult to solve explicitly. Hitchin [Hi3,Hi2] constructed some solutions of Painlevé VI by relating certain isomonodromic deformations to equivariant twistor geometry. The prototype is the irreducible linear $S L_{2}(\mathbb{C})$ action on $\mathbb{C} P^{3}$, which gives rise to a natural flat meromorphic connection on $\mathbb{C} P^{3}$. The restriction to an embedded line $\mathbb{C} P^{1}$ is isomonodromic under deformation of the line. The corresponding Frobenius manifold is the $n=0$ instance of Theorem 3.1.

New isomonodromic transformations can in principle be constructed by applying "Schlesinger transformations", which are meromorphic gauge transformations on $\mathbb{C} P^{1}[\mathrm{JM}]$. We refer to the recent paper of Manin [Mn2] for a discussion of the corresponding transformations of Painlevé VI solutions. Equivariant twistor geometry provides a method for constructing some Schlesinger transformations explicitly. The prototype $\mathbb{C} P^{3}$ with the irreducible $S L_{2}(\mathbb{C})$ action is the equivariant twistor space of the Riemannian manifold $S^{4}$ with a certain isometric $S U_{2}$ action. The Atiyah-Ward correspondence [At] relates anti-selfdual Yang-Mills instantons on $S^{4}$ to certain holomorphic bundles on the twistor space $\mathbb{C} P^{3}$. An equivariant version relates instantons with $S U_{2}$ symmetry to holomorphic bundles with $S L_{2}(\mathbb{C})$ symmetry. These equivariant objects were constructively classified in [BS] by an equivariant version of the ADHM method [ADHM]. Isomonodromic deformations can be generated from the equivariant ADHM monads, and all are related by Schlesinger transformations. The Frobenius manifolds of Theorem 3.1 are constructed from the equivariant ADHM data.

I would like to thank G. Bor and N.J. Hitchin for helping me in understanding some of these topics.

\section{Semisimple Frobenius manifolds}

We define semisimple (or massive) Frobenius manifolds in the framework of local canonical coordinates [D1,Hi1,Mn1], and state some basic results for threedimensional Frobenius manifolds. All coordinates are complex, all functions are holomorphic, and all derivatives are with respect to a complex variable. We follow the notational conventions of [CDD; Sec. VI.A.4] for the exterior derivative $d$, Lie derivative $\mathcal{L}_{V}$, and interior product $i_{V}$. Riemannian metrics are complex bilinear, not hermitian.

We first introduce the Euler vector field and the identity vector field. The complex Lie group $S L_{2}(\mathbb{C})$ acts on the Riemann sphere $\mathbb{C} \cup\{\infty\}$ by fractional linear transformations. The two-dimensional Borel subgroup $B \subset S L_{2}(\mathbb{C})$ consisting of the upper-triangular matrices is the stabilizer of $\infty$. $B$ acts by dilations and translations, $x \mapsto a x+b$, on the coordinates of a point $x \in \mathbb{C}$. Using the notation $\partial=\frac{\partial}{\partial x}$, the dilations are generated by the "Euler vector field" $E=x \partial$ and the translations by the "identity vector field" $I=\partial$, with $[I, E]=I$. More 
generally, for the action of $B$ on the coordinates $\left(x_{1}, x_{2}, \ldots, x_{n}\right) \in \mathbb{C}^{n}$ of an $n$ tuple of points in $\mathbb{C}$, the Euler vector field generating the dilations is the radial vector field

$$
E=x_{1} \partial_{1}+x_{2} \partial_{2}+\cdots+x_{n} \partial_{n},
$$

and the identity vector field generating the translations is

$$
I=\partial_{1}+\partial_{2}+\cdots+\partial_{n}
$$

where $[I, E]=I$ as before. Note that the Euler vector field is characterized by the property $\mathcal{L}_{E} x_{i}=x_{i}$, and the identity vector field by the property $\mathcal{L}_{I} x_{i}=1$. A function $f$ on $\mathbb{C}^{n}$, or on an open subset $U \subset \mathbb{C}^{n}$, will be called " $B$-invariant" if $\mathcal{L}_{E} f=0$ and $\mathcal{L}_{I} f=0$. We will say that a function $f$ is of "homogeneity $m$ " if $\mathcal{L}_{E} f=m f$ for a constant $m$.

A Riemannian metric $g$ is "flat" if the curvature of the associated Levi-Civita connection $\nabla$ is zero. A Riemannian metric on $U \subset \mathbb{C}^{n}$ is "diagonal" if it is of the form

$$
g=g_{11} d x_{1} \otimes d x_{1}+g_{22} d x_{2} \otimes d x_{2}+\cdots+g_{n n} d x_{n} \otimes d x_{n}
$$

Definition 2.1. A "semisimple Frobenius manifold" structure of homogeneity $m$ on open subset $U \subset \mathbb{C}^{n}$ with "canonical coordinates" $\left(x_{1}, x_{2}, \ldots, x_{n}\right)$ consists of a diagonal metric $g$ satisfying the three conditions:

(M1) $g$ is flat.

(M2) The components $g_{i i}$ are functions of homogeneity $m$.

(M3) The identity vector field $I$ is covariantly constant with respect to the Levi-Civita connection.

A Frobenius metric is "nontrivial" if the components $g_{i i}$ are not all constant; the standard Euclidean metric is an example of a trivial Frobenius metric. Now apply definition 2.1 to an atlas of local coordinate charts on a manifold. A complex manifold $M$ with a complex metric $g$ and vector fields $E$ and $I$ satisfying $[I, E]=I$ is a "semisimple Frobenius manifold" of homogeneity $m$ if every point of $M$ has a neighborhood which admits local canonical coordinates $\left(x_{1}, x_{2}, . ., x_{n}\right)$ as above. It is convenient to relax this definition, requiring only that every point on some dense open subset has such a neighborhood, and that $g$ is nondegenerate and nonsingular only on some dense open subset.

We now focus on the three-dimensional case. The cross-ratio

$$
t=\frac{x_{3}-x_{1}}{x_{2}-x_{1}}
$$

is a $B$-invariant function on $\mathbb{C}^{3}$. In section 4 , we will prove: 
Proposition 2.2. The metric $g=g_{11} d x_{1} \otimes d x_{1}+g_{22} d x_{2} \otimes d x_{2}+g_{33} d x_{3} \otimes d x_{3}$ is a homogeneity-0 Frobenius metric if and only if

$$
t d g_{11}=(1-t) d g_{22}=t(t-1) d g_{33}=2 c \sqrt{g_{11} g_{22} g_{33}} d t
$$

for some constant $c$. The Frobenius metric is nontrivial if and only if $c$ is nonzero.

It is evident that such a $c$ is unique, and that the "trace"

$$
k=-\frac{c^{2}}{2}\left(g_{11}+g_{22}+g_{33}\right)
$$

of a homogeneity-0 Frobenius metric $g$ is a constant. The trace is unchanged under the rescaling $g \mapsto \alpha g$ by a constant $\alpha$.

The two-dimensional Lie group $B$ is a symmetry group of a three-dimensional Frobenius metrics. For a nontrivial homogeneity-0 Frobenius metric, $i_{Y}$ applied to eq. (2.6) yields

$$
t \mathcal{L}_{Y} g_{11}=(1-t) \mathcal{L}_{Y} g_{22}=t(t-1) \mathcal{L}_{Y} g_{33}=2 c \sqrt{g_{11} g_{22} g_{33}} \mathcal{L}_{Y} t
$$

for any vector field $Y$. The $B$-invariance of $t, \mathcal{L}_{E} t=\mathcal{L}_{I} t=0$, then implies the $B$-invariance of $g_{i i}, \mathcal{L}_{E} g_{i i}=\mathcal{L}_{I} g_{i i}=0$. More generally for homogeneity $m$, condition (M2) states $\mathcal{L}_{E} g_{i i}=m g_{i i}$, and the results of section 4 give $\mathcal{L}_{I} g_{i i}=0$. In the language of Riemannian geometry, $I$ is a Killing vector, $\mathcal{L}_{I} g=0$, and $E$ is a weight- $(m+2)$ conformal Killing vector, $\mathcal{L}_{E} g=(m+2) g$.

\section{Frobenius manifolds constructed from instantons}

We start by constructing an atlas of canonical coordinate charts, and the corresponding Euler and identity vector fields, on a certain hypersurface $M \subset$ $\mathbb{C}^{4}$. Let $\left(z_{1}, z_{2}, z_{3}, r\right)$ be the linear coordinates on $\mathbb{C}^{4}$, let $M$ be the hypersurface defined by the vanishing of

$$
Q=\left(z_{1}-z_{2}\right)^{2}+\left(z_{2}-z_{3}\right)^{2}+\left(z_{3}-z_{1}\right)^{2}-2 r^{2},
$$

and let $j: M \rightarrow \mathbb{C}^{4}$ denote the inclusion map. Every point in an open dense subset of $M$ has a neighborhood on which the restrictions $\left(x_{1}, x_{2}, x_{3}\right)=$ $\left(j^{*} q_{1}, j^{*} q_{2}, j^{*} q_{3}\right)$ of the three functions

$$
\begin{aligned}
& q_{1}=2 r\left(2 z_{1}-z_{2}-z_{3}\right)-3 z_{1}^{2}-6 z_{2} z_{3}, \\
& q_{2}=2 r\left(2 z_{2}-z_{3}-z_{1}\right)-3 z_{2}^{2}-6 z_{3} z_{1}, \\
& q_{3}=2 r\left(2 z_{3}-z_{1}-z_{2}\right)-3 z_{3}^{2}-6 z_{1} z_{2},
\end{aligned}
$$

define local coordinates; the points which fail to have this property are characterized by the vanishing of the Jacobian determinant. Of course these coordinates 
are only valid locally because the functions $x_{i}: M \rightarrow \mathbb{C}$ are not one-to-one. The group of dilations on $\mathbb{C}^{4}$ is generated by the radial vector field $2 \tilde{E}$, where

$$
\tilde{E}=\frac{1}{2}\left(z_{1} \frac{\partial}{\partial z_{1}}+z_{2} \frac{\partial}{\partial z_{2}}+z_{3} \frac{\partial}{\partial z_{3}}+r \frac{\partial}{\partial r}\right)
$$

Now $\tilde{E}$ is tangent to $M$ since $\mathcal{L}_{\tilde{E}} Q=Q$, so the restriction of $\tilde{E}$ projects to a vector field $E$ on $M$. The vector field $E$ is the Euler vector field relative to the local coordinates since $\mathcal{L}_{\tilde{E}} q_{i}=q_{i}$. The vector field

$$
\tilde{I}=\frac{-1}{6\left(z_{1}+z_{2}+z_{3}\right)}\left(\frac{\partial}{\partial z_{1}}+\frac{\partial}{\partial z_{2}}+\frac{\partial}{\partial z_{3}}\right)
$$

is tangent to $M$ since $\mathcal{L}_{\tilde{I}} Q=0$, so the restriction of $\tilde{I}$ projects to a vector field $I$ on $M$. The vector field $I$ is the identity vector field relative to the local coordinates since $\mathcal{L}_{\tilde{I}} q_{i}=1$, which follows from the identity $q_{1}+q_{2}+q_{3}=$ $-3\left(z_{1}+z_{2}+z_{3}\right)^{2}$. Let the symmetric group $S_{3}$ act on $\mathbb{C}^{4}$ by permuting the first three coordinates $\left(z_{1}, z_{2}, z_{3}\right)$. The polynomial $Q$ is $S_{3}$-invariant, so the action maps the hypersurface $M$ to itself. The $S_{3}$ action permutes the three functions $q_{i}$, leaving the vector fields $\tilde{E}$ and $\tilde{I}$ invariant.

Our main result is the existence of a family of Frobenius metrics on $M$, the coefficients of which are rational functions on $\mathbb{C}^{4}$ :

Theorem 3.1. For each nonnegative integer $n$, there exist triplets $\left(b_{1}, b_{2}, b_{3}\right)$ and $\left(u_{1}, u_{2}, u_{3}\right)$ of explicitly computable homogeneous polynomials of degree $l \leq$ $2\left(n^{2}+n+2\right)$ on $\mathbb{C}^{4}$ such that

$$
g=j^{*}\left(\frac{u_{1}}{b_{1}} d q_{1} \otimes d q_{1}+\frac{u_{2}}{b_{2}} d q_{2} \otimes d q_{2}+\frac{u_{3}}{b_{3}} d q_{3} \otimes d q_{3}\right)
$$

is the metric of homogeneity-0 Frobenius manifold on the hypersurface $M \subset \mathbb{C}^{4}$. The Frobenius metric $g$ is nontrivial, and has trace $k=\frac{1}{2}\left(n+\frac{1}{2}\right)^{2}$. The symmetric group $S_{3}$ acts by permutation on each of the triplets $\left(b_{1}, b_{2}, b_{3}\right),\left(u_{1}, u_{2}, u_{3}\right)$, and $\left(q_{1}, q_{2}, q_{3}\right)$, so the Frobenius structure on $M$ is $S_{3}$-invariant.

The constructive geometric proof of theorem 3.1 for all nonnegative integers $n$, using the classification by Bor and the author [BS] of Yang-Mills instantons with a certain $S U_{2}$-symmetry, will be described elsewhere. In the present paper, we exhibit the polynomials $b_{i}$ and $u_{i}$ for $n \leq 2$, and compute the canonical coordinate expressions of the Frobenius metrics. Applying Proposition 2.2 to these expressions constitutes a computational proof of theorem 3.1 for $n \leq 2$. We do not continue beyond $n=2$ because the size of the expressions grows very quickly with $n$.

The local canonical coordinate expressions of the form eq.(2.3) are easily evaluated for the Frobenius metrics eq.(3.1). We first observe that a $B$-invariant 
function on $U \subset \mathbb{C}^{3}$ depends only on the cross-ratio $t$. However, a $B$-invariant function on $M$ is a possibly multi-valued function of $t$, because the cross ratio $t: M \rightarrow \mathbb{C}$, although well-defined, is not one-to-one. If a path $\gamma: \mathbb{C} \rightarrow M$ is transverse to $B$-orbits, then it follows from Theorem 3.1 and $B$-invariance that

$$
g_{i i}\left(x_{1}, x_{2}, x_{3}\right)=\frac{u_{i}(\gamma(w))}{b_{i}(\gamma(w))},
$$

where $w$ is a (possibly non-unique) solution of

$$
\frac{x_{3}-x_{1}}{x_{2}-x_{1}}=\frac{q_{3}(\gamma(w))-q_{1}(\gamma(w))}{q_{2}(\gamma(w))-q_{1}(\gamma(w))} .
$$

Choosing the polynomial path

$$
\gamma(w)=\left(w^{2}-1,-2 w+2,2 w+2, w^{2}+3\right) \in M \subset \mathbb{C}^{4},
$$

the $g_{i i}$ become rational functions of degree at most $2 l$ in the variable $w$, where $w$ is a solution of

$$
\frac{x_{3}-x_{1}}{x_{2}-x_{1}}=\frac{(w+1)(w-3)^{3}}{(w-1)(w+3)^{3}} .
$$

In terms of the cross-ratio $t$ of the local coordinates $\left(x_{1}, x_{2}, x_{3}\right), w$ is a root of the following quartic polynomial with coefficients depending on $t$ :

$$
(w-1)(w+3)^{3} t-(w+1)(w-3)^{3}=0 .
$$

We recall that the roots of a quartic polynomial can be expressed as explicit (multivalued) algebraic function of the coefficients by a formula analogous to the familiar quadratic formula, albeit much more complicated (see e.g. [Ro]).

We now exhibit the data of theorem 3.1 for the first few values of $n$. For $n=0$, the data is constructed from the ADHM monad of the trivial Yang-Mills instanton. The trivial instanton has instanton number 0 , and its symmetry group contains the $\mathrm{SU}_{2}$ symmetry group of [BS]. The homogeneous polynomials $u_{i}$ and $b_{i}$ on $\mathbb{C}^{4}$ have degree $l=2$ :

$$
\begin{array}{ll}
b_{1}=36\left(z_{3}-z_{1}\right)\left(z_{1}-z_{2}\right), & u_{1}=\left(r-\left(z_{3}-z_{1}\right)+\left(z_{1}-z_{2}\right)\right)^{2}, \\
b_{2}=36\left(z_{1}-z_{2}\right)\left(z_{2}-z_{3}\right), & u_{2}=\left(r-\left(z_{1}-z_{2}\right)+\left(z_{2}-z_{3}\right)\right)^{2}, \\
b_{3}=36\left(z_{2}-z_{3}\right)\left(z_{3}-z_{1}\right), & u_{3}=\left(r-\left(z_{2}-z_{3}\right)+\left(z_{3}-z_{1}\right)\right)^{2} .
\end{array}
$$

The $S_{3}$ symmetry of the triplet $\left(b_{1}, b_{2}, b_{3}\right)$ and of the triplet $\left(u_{1}, u_{2}, u_{3}\right)$ is evident. The canonical coordinate expression of the $n=0$ metric is

$$
\begin{aligned}
g= & -\frac{(w-1)(w+1)}{4(w-3)(w+3)} d x_{1} \otimes d x_{1}-\frac{(w-1)}{4 w(w+3)} d x_{2} \otimes d x_{2} \\
& +\frac{(w+1)}{4 w(w-3)} d x_{3} \otimes d x_{3},
\end{aligned}
$$


where $w$ is related to the cross-ratio $t$ by eq.(3.2). The reader may easily check that this metric satisfies the conditions of Proposition 2.2 with $c=1$, and has trace $k=\frac{1}{8}$.

For $n=1$, the data is constructed from the ADHM monad of the "basic instanton" [At], which is the unique $S U_{2}$-symmetric instanton with instanton number 1 . The homogeneous polynomials $u_{i}$ and $b_{i}$ on $\mathbb{C}^{4}$ have degree $l=6$ :

$$
b_{1}=216 r^{2}\left(z_{2}-z_{3}\right)^{2}\left(z_{3}-z_{1}\right)\left(z_{1}-z_{2}\right),
$$

and $u_{1}$ is an irreducible polynomial with 84 terms :

$$
\begin{aligned}
& u_{1}=-3 r^{6}+14 r^{5} z_{1}-21 r^{4} z_{1}{ }^{2}+4 r^{3} z_{1}{ }^{3}+19 r^{2} z_{1}{ }^{4}-18 r z_{1}{ }^{5}+5 z_{1}{ }^{6}-7 r^{5} z_{2} \\
& +21 r^{4} z_{1} z_{2}-6 r^{3} z_{1}{ }^{2} z_{2}-38 r^{2} z_{1}{ }^{3} z_{2}+45 r z_{1}{ }^{4} z_{2}-15 z_{1}{ }^{5} z_{2}+39 r^{4} z_{2}{ }^{2} \\
& +108 r^{3} z_{1} z_{2}{ }^{2}+240 r^{2} z_{1}{ }^{2} z_{2}{ }^{2}+66 r z_{1}{ }^{3} z_{2}{ }^{2}+33 z_{1}{ }^{4} z_{2}{ }^{2}-53 r^{3} z_{2}{ }^{3} \\
& -221 r^{2} z_{1} z_{2}{ }^{3}-144 r z_{1}{ }^{2} z_{2}{ }^{3}-41 z_{1}{ }^{3} z_{2}{ }^{3}+22 r^{2} z_{2}{ }^{4}+27 r z_{1} z_{2}{ }^{4}-33 z_{1}{ }^{2} z_{2}{ }^{4} \\
& +12 r z_{2}{ }^{5}+51 z_{1} z_{2}{ }^{5}-10 z_{2}{ }^{6}-7 r^{5} z_{3}+21 r^{4} z_{1} z_{3}-6 r^{3} z_{1}{ }^{2} z_{3}-38 r^{2} z_{1}{ }^{3} z_{3} \\
& +45 r z_{1}^{4} z_{3}-15 z_{1}^{5} z_{3}-99 r^{4} z_{2} z_{3}-204 r^{3} z_{1} z_{2} z_{3}-366 r^{2} z_{1}^{2} z_{2} z_{3} \\
& -312 r z_{1}^{3} z_{2} z_{3}+9 z_{1}{ }^{4} z_{2} z_{3}+51 r^{3} z_{2}^{2} z_{3}+183 r^{2} z_{1} z_{2}^{2} z_{3} \\
& +234 r z_{1}^{2} z_{2}{ }^{2} z_{3}-9 z_{1}{ }^{3} z_{2}{ }^{2} z_{3}+133 r^{2} z_{2}{ }^{3} z_{3}+180 r z_{1} z_{2}{ }^{3} z_{3}+255 z_{1}{ }^{2} z_{2}{ }^{3} z_{3} \\
& -87 r z_{2}{ }^{4} z_{3}-189 z_{1} z_{2}{ }^{4} z_{3}+9 z_{2}{ }^{5} z_{3}+39 r^{4} z_{3}{ }^{2}+108 r^{3} z_{1} z_{3}{ }^{2}+240 r^{2} z_{1}{ }^{2} z_{3}{ }^{2} \\
& +66 r z_{1}^{3} z_{3}{ }^{2}+33 z_{1}{ }^{4} z_{3}{ }^{2}+51 r^{3} z_{2} z_{3}{ }^{2}+183 r^{2} z_{1} z_{2} z_{3}{ }^{2}+234 r z_{1}{ }^{2} z_{2} z_{3}{ }^{2} \\
& -9 z_{1}{ }^{3} z_{2} z_{3}{ }^{2}-291 r^{2} z_{2}{ }^{2} z_{3}{ }^{2}-504 r z_{1} z_{2}{ }^{2} z_{3}{ }^{2}-369 z_{1}{ }^{2} z_{2}{ }^{2} z_{3}{ }^{2}+84 r z_{2}{ }^{3} z_{3}{ }^{2} \\
& +123 z_{1} z_{2}{ }^{3} z_{3}{ }^{2}+72 z_{2}{ }^{4} z_{3}{ }^{2}-53 r^{3} z_{3}{ }^{3}-221 r^{2} z_{1} z_{3}{ }^{3}-144 r z_{1}{ }^{2} z_{3}{ }^{3}-41 z_{1}{ }^{3} z_{3}{ }^{3} \\
& +133 r^{2} z_{2} z_{3}{ }^{3}+180 r z_{1} z_{2} z_{3}{ }^{3}+255 z_{1}{ }^{2} z_{2} z_{3}{ }^{3}+84 r z_{2}{ }^{2} z_{3}{ }^{3}+123 z_{1} z_{2}{ }^{2} z_{3}{ }^{3} \\
& -137 z_{2}{ }^{3} z_{3}{ }^{3}+22 r^{2} z_{3}{ }^{4}+27 r z_{1} z_{3}{ }^{4}-33 z_{1}{ }^{2} z_{3}{ }^{4}-87 r z_{2} z_{3}{ }^{4}-189 z_{1} z_{2} z_{3}{ }^{4} \\
& +72 z_{2}{ }^{2} z_{3}{ }^{4}+12 r z_{3}{ }^{5}+51 z_{1} z_{3}{ }^{5}+9 z_{2} z_{3}{ }^{5}-10 z_{3}{ }^{6} \text {. }
\end{aligned}
$$

The canonical coordinate expression of the $n=1$ metric is

$$
\begin{aligned}
g= & -\frac{9(w-1)^{3}(w+1)^{3}}{4(w-3)(w+3)\left(w^{2}+3\right)^{2}} d x_{1} \otimes d x_{1}-\frac{9(w-1)^{3}}{w(w+3)\left(w^{2}+3\right)^{2}} d x_{2} \otimes d x_{2} \\
& +\frac{9(w+1)^{3}}{w(w-3)\left(w^{2}+3\right)^{2}} d x_{3} \otimes d x_{3} .
\end{aligned}
$$

This metric satisfies the conditions of Proposition 2.2 with $c=1$, and has trace $k=\frac{9}{8}$.

For $n=2$, the data is constructed from the ADHM monad of the unique $S U_{2^{-}}$ symmetric instanton $[\mathrm{BS}]$ with instanton number 3 . The homogeneous polynomials $u_{i}$ and $b_{i}$ on $\mathbb{C}^{4}$ have degree $l=10$ :

$$
\begin{aligned}
b_{1}= & 36\left(z_{2}-z_{3}\right)^{2}\left(z_{3}-z_{1}\right)\left(z_{1}-z_{2}\right)\left(52 r^{3}+2 z_{1}{ }^{3}-3 z_{1}{ }^{2} z_{2}-3 z_{1} z_{2}{ }^{2}\right. \\
& \left.+2 z_{2}{ }^{3}-3 z_{1}{ }^{2} z_{3}+12 z_{1} z_{2} z_{3}-3 z_{2}{ }^{2} z_{3}-3 z_{1} z_{3}{ }^{2}-3 z_{2} z_{3}{ }^{2}+2 z_{3}{ }^{3}\right)^{2},
\end{aligned}
$$


and $u_{1}$ is an irreducible polynomial with 283 terms, of which we only exhibit the first few:

$$
\begin{aligned}
u_{1} & =25\left(174 r^{10}-764 r^{9} z_{1}+1122 r^{8} z_{1}{ }^{2}-244 r^{7} z_{1}{ }^{3}-998 r^{6} z_{1}{ }^{4}+1008 r^{5} z_{1}{ }^{5}\right. \\
& -274 r^{4} z_{1}{ }^{6}-20 r^{3} z_{1}{ }^{7}-24 r^{2} z_{1}{ }^{8}+20 r z_{1}{ }^{9}+382 r^{9} z_{2}-1122 r^{8} z_{1} z_{2} \\
& \left.+366 r^{7} z_{1}{ }^{2} z_{2}+1996 r^{6} z_{1}{ }^{3} z_{2}-2520 r^{5} z_{1}{ }^{4} z_{2}+822 r^{4} z_{1}{ }^{5} z_{2}+\cdots \cdots\right) .
\end{aligned}
$$

The canonical coordinate expression of the $n=2$ metric is

$$
\begin{aligned}
g= & -\frac{25(w-1)^{5}(w+1)^{5}\left(w^{2}+5\right)^{2}}{4(w-3)(w+3)\left(w^{2}+1\right)^{2}\left(w^{2}-2 w+5\right)^{2}\left(w^{2}+2 w+5\right)^{2}} d x_{1} \otimes d x_{1} \\
& -\frac{25(w-1)^{5}\left(3 w^{2}+2 w+7\right)^{2}}{w(w+3)\left(w^{2}+1\right)^{2}\left(w^{2}-2 w+5\right)^{2}\left(w^{2}+2 w+5\right)^{2}} d x_{2} \otimes d x_{2} \\
& +\frac{25(w+1)^{5}\left(3 w^{2}-2 w+7\right)^{2}}{w(w-3)\left(w^{2}+1\right)^{2}\left(w^{2}-2 w+5\right)^{2}\left(w^{2}+2 w+5\right)^{2}} d x_{3} \otimes d x_{3} .
\end{aligned}
$$

This metric satisfies the conditions of Proposition 2.2 with $c=1$, and has trace $k=\frac{25}{8}$. For reasons of brevity, we do not continue beyond $n=2$.

Corollary 4.3 below associates a pair of Frobenius metrics $g^{ \pm}$of homogeneity $m= \pm \sqrt{8 k}$ to each Frobenius metric $g$ of homogeneity 0 and trace $k$. The metrics $g^{ \pm}$associated to the $n=0$ metric of Theorem 3.1 can be expressed in the form eq.(3.1). The homogeneous polynomials $u_{i}^{+}$have degree 4 ,

$$
u_{1}^{+}=\left(r^{2}+4 r z_{1}-5 z_{1}^{2}-2 r z_{2}+5 z_{1} z_{2}+z_{2}^{2}-2 r z_{3}+5 z_{1} z_{3}-7 z_{2} z_{3}+z_{3}^{2}\right)^{2},
$$

the polynomials $u_{i}^{-}$have degree $0, u_{1}^{-}=1$, and the $b_{i}^{ \pm}$are equal to the degree 2 polynomials $b_{i}$ of eq.(3.4). The canonical coordinate expressions for the homogeneity $m= \pm 1$ metrics are

$$
\begin{gathered}
g^{+}=-\frac{4(w+1)}{(w-3)(w+3)^{4}}\left(x_{1}-x_{2}\right) d x_{1} \otimes d x_{1}-\frac{(w+1)^{4}}{4 w(w+3)^{4}}\left(x_{1}-x_{2}\right) d x_{2} \otimes d x_{2} \\
+\frac{(w-1)^{3}(w+1)}{4 w(w-3)(w+3)^{3}}\left(x_{1}-x_{2}\right) d x_{3} \otimes d x_{3} \\
g^{-}=-\frac{(w+3)^{2}}{(w-3)(w+1)}\left(x_{1}-x_{2}\right)^{-1} d x_{1} \otimes d x_{1}-\frac{(w+3)^{2}}{4 w}\left(x_{1}-x_{2}\right)^{-1} d x_{2} \otimes d x_{2} \\
+\frac{(w-1)(w+3)^{3}}{4 w(w-3)(w+1)}\left(x_{1}-x_{2}\right)^{-1} d x_{3} \otimes d x_{3} .
\end{gathered}
$$

The canonical coordinate expressions for the Frobenius metrics of homogeneity $m= \pm 3, \pm 5, \ldots$ associated to $n=1,2, \ldots$ can also be computed explicitly, but I do not know whether these metrics can be expressed in the form eq.(3.1). 
Applying the correspondence between semisimple three-dimensional Frobenius manifolds and Painlevé transcendents $[\mathrm{D} 1, \mathrm{Hi1}, \mathrm{JM}]$ to the Frobenius manifolds of theorem 3.1, we obtain for each nonnegative integer $n$ two distinct solutions $\lambda_{ \pm}(t)$ of the Painlevé VI equation

$$
\begin{aligned}
\frac{d^{2} \lambda_{ \pm}}{d t^{2}}= & \frac{1}{2}\left(\frac{1}{\lambda_{ \pm}}+\frac{1}{\lambda_{ \pm}-1}+\frac{1}{\lambda_{ \pm}-t}\right)\left(\frac{d \lambda_{ \pm}}{d t}\right)^{2}-\left(\frac{1}{t}+\frac{1}{t-1}+\frac{1}{\lambda_{ \pm}-t}\right) \frac{d \lambda_{ \pm}}{d t} \\
& +\frac{\lambda_{ \pm}\left(\lambda_{ \pm}-1\right)\left(\lambda_{ \pm}-t\right)}{t^{2}(t-1)^{2}}\left(\alpha_{ \pm}+\beta \frac{t}{\lambda_{ \pm}^{2}}+\gamma \frac{t-1}{\left(\lambda_{ \pm}-1\right)^{2}}+\delta \frac{t(t-1)}{\left(\lambda_{ \pm}-t\right)^{2}}\right)
\end{aligned}
$$

with

$\alpha_{ \pm}=\frac{1}{2}\left(\left(n+\frac{1}{2}\right) \mp 1\right)^{2}, \quad \beta=-\frac{1}{2}\left(n+\frac{1}{2}\right)^{2}, \quad \gamma=\frac{1}{2}\left(n+\frac{1}{2}\right)^{2}, \quad \delta=\frac{1}{2}-\frac{1}{2}\left(n+\frac{1}{2}\right)^{2}$.

The solution $\lambda_{ \pm}$is a rational function of degree $l_{ \pm} \leq 2\left(n^{2}+n+2\right)$ in the variable $w$, where $w$ is related to $t$ as in eq.(3.2) above. The explicit formulae for $\lambda_{ \pm}$are exhibited in [Se] for $n \leq 4$, building on Hitchin's previous computation [Hi3] of $\lambda_{+}$for $n=0$.

\section{Frobenius coframes in dimension three}

An "orthonormal frame" for a Riemannian metric $g$ is a triplet of vector fields $e_{i}$ such that $g\left(e_{i}, e_{j}\right)=\delta_{i, j}$. The dual "orthonormal coframe" is the triplet of one-forms $\theta_{j}$ such that $\left\langle\theta_{i}, e_{j}\right\rangle=\delta_{i, j}$. The metric can be reconstructed from an orthonormal coframe by $g=\theta_{1} \otimes \theta_{1}+\theta_{2} \otimes \theta_{2}+\theta_{3} \otimes \theta_{3}$. We will follow the notational conventions of [CDD; Sec. VI.B].

A linear connection $\nabla$ on the tangent bundle which preserves the metric is an "orthogonal" connection. Relative to an orthonormal frame, an orthogonal connection $\nabla$ is expressed in terms of the triplet of connection one-forms $\Omega_{k}$ as follows: If $X=X_{1} e_{1}+X_{2} e_{2}+X_{3} e_{3}$, then $\nabla X=(\nabla X)_{1} \otimes e_{1}+(\nabla X)_{2} \otimes e_{2}+$ $(\nabla X)_{3} \otimes e_{3}$, where

$$
\begin{aligned}
& (\nabla X)_{1}=d X_{1}-\Omega_{2} X_{3}+\Omega_{3} X_{2} \\
& (\nabla X)_{2}=d X_{2}-\Omega_{3} X_{1}+\Omega_{1} X_{3} \\
& (\nabla X)_{3}=d X_{3}-\Omega_{1} X_{2}+\Omega_{2} X_{1} .
\end{aligned}
$$

using the three-vector notation

$$
\vec{X}=\left(\begin{array}{c}
X_{1} \\
X_{2} \\
X_{3}
\end{array}\right), \quad \vec{\Omega}=\left(\begin{array}{c}
\Omega_{1} \\
\Omega_{2} \\
\Omega_{3}
\end{array}\right), \quad \vec{\theta}=\left(\begin{array}{c}
\theta_{1} \\
\theta_{2} \\
\theta_{3}
\end{array}\right),
$$

and the cross-product $\times$, eq.(4.1) becomes

$$
\vec{\nabla} \vec{X}=d \vec{X}-\vec{\Omega} \times \vec{X} .
$$


The Levi-Civita connection of $g$ is the unique orthogonal connection with zero torsion. If $\vec{\theta}$ is an orthonormal coframe for $g$, then the connection form $\vec{\Omega}$ corresponding to the Levi-Civita connection is the unique solution of the Cartan torsion equation

$$
d \vec{\theta}-\vec{\Omega} \times \vec{\theta}=0 .
$$

We will say that $\vec{\Omega}$ is the "Levi-Civita connection form" of the orthonormal coframe $\vec{\theta}$ if the Cartan torsion equation holds. The curvature of the Levi-Civita connection is encoded in the Cartan curvature two-forms

$$
\vec{R}=d \vec{\Omega}-\frac{1}{2} \vec{\Omega} \times \vec{\Omega}
$$

and the metric is flat if and only if $\vec{R}=0$.

On $U \subset \mathbb{C}^{3}$ with coordinates $\left(x_{1}, x_{2}, x_{3}\right)$, we say that a triplet of one-forms $\vec{\theta}$ is a "canonical coframe" if there is a triplet $\vec{H}$ of functions such that

$$
\vec{H}=\left(\begin{array}{c}
h_{1} \\
h_{2} \\
h_{3}
\end{array}\right), \quad \vec{\theta}=\left(\begin{array}{c}
\theta_{1} \\
\theta_{2} \\
\theta_{3}
\end{array}\right)=\left(\begin{array}{c}
h_{1} d x_{1} \\
h_{2} d x_{2} \\
h_{1} d x_{3}
\end{array}\right), \quad \vec{e}=\left(\begin{array}{c}
e_{1} \\
e_{2} \\
e_{3}
\end{array}\right)=\left(\begin{array}{c}
h_{1}^{-1} \partial_{1} \\
h_{2}^{-1} \partial_{2} \\
h_{3}^{-1} \partial_{3}
\end{array}\right),
$$

where $\vec{e}$ is the orthonormal frame dual to $\vec{\theta}$. A canonical coframe can be reconstructed from its "canonical coefficients" $\vec{H}=i_{I} \vec{\theta}$. Obviously a metric $g$ admits an orthonormal canonical coframe if and only if $g$ is diagonal as in eq.(2.3). We define a homogeneity- $m$ "Frobenius coframe" to be a canonical coframe $\vec{\theta}$ such that

$$
g=\theta_{1} \otimes \theta_{1}+\theta_{2} \otimes \theta_{2}+\theta_{3} \otimes \theta_{3}=h_{1}^{2} d x_{1} \otimes d x_{1}+h_{2}^{2} d x_{2} \otimes d x_{2}+h_{3}^{2} d x_{3} \otimes d x_{3}
$$

is a homogeneity- $m$ Frobenius metric.

We say that a connection form $\vec{\Omega}$ is "Egoroff" if there is a triplet $\vec{F}$ of functions such that

$$
\vec{F}=\left(\begin{array}{c}
f_{1} \\
f_{2} \\
f_{3}
\end{array}\right), \quad \vec{\Omega}=\left(\begin{array}{l}
\Omega_{1} \\
\Omega_{2} \\
\Omega_{3}
\end{array}\right)=\left(\begin{array}{l}
f_{1}\left(x_{2}-x_{3}\right)^{-1}\left(d x_{2}-d x_{3}\right) \\
f_{2}\left(x_{3}-x_{1}\right)^{-1}\left(d x_{3}-d x_{1}\right) \\
f_{3}\left(x_{1}-x_{2}\right)^{-1}\left(d x_{1}-d x_{2}\right)
\end{array}\right)
$$

An Egoroff connection form can be reconstructed from the "Egoroff coefficients" $\vec{F}=i_{E} \vec{\Omega}$. This is a nonstandard definition, but in Lemma A.3 we'll show that the Levi-Civita connection form $\vec{\Omega}$ of an orthonormal canonical coframe is Egoroff if and only if the metric $g$ is Egoroff in the standard sense.

The following proposition, which is proved in the Appendix, simultaneously characterizes three-dimensional Frobenius coframes and their Levi-Civita connection forms: 
Proposition 4.1. Let $\vec{\theta}$ be a canonical coframe. Then $\vec{\theta}$ is a homogeneity-m Frobenius coframe with Levi-Civita connection form $\vec{\Omega}$ if and only if the following four conditions hold:

(C1) $\vec{\Omega}$ is an Egoroff connection form.

(C2) $d \vec{F}-\vec{\Omega} \times \vec{F}=0$, where $\vec{F}=i_{E} \vec{\Omega}$.

(C3) $d \vec{H}-\vec{\Omega} \times \vec{H}=0$, where $\vec{H}=i_{I} \vec{\theta}$.

(C4) $\vec{F} \times \vec{H}=\frac{m}{2} \vec{H}$.

The remainder of this section is based on corollaries of Proposition 4.1.

The following differential equation for $\vec{F}$ will be called the "structural equation":

$$
\begin{aligned}
& d f_{1}-f_{2} f_{3}\left(\frac{d x_{3}-d x_{1}}{x_{3}-x_{1}}-\frac{d x_{1}-d x_{2}}{x_{1}-x_{2}}\right)=0, \\
& d f_{2}-f_{3} f_{1}\left(\frac{d x_{1}-d x_{2}}{x_{1}-x_{2}}-\frac{d x_{2}-d x_{3}}{x_{2}-x_{3}}\right)=0 \\
& d f_{3}-f_{1} f_{2}\left(\frac{d x_{2}-d x_{3}}{x_{2}-x_{3}}-\frac{d x_{3}-d x_{1}}{x_{3}-x_{1}}\right)=0 .
\end{aligned}
$$

If $\vec{\theta}$ is a Frobenius coframe with Levi-Civita connection form $\vec{\Omega}$, then conditions (C1) and (C2) of Proposition 4.1 are equivalent to the statement that the Egoroff coefficients $\vec{F}=i_{E} \vec{\Omega}$ solve the structural equation. The following corollary of Proposition 4.1 is only slightly less obvious.

Corollary 4.2. A canonical coframe $\vec{\theta}$ is a nontrivial homogeneity-0 Frobenius coframe if and only if some nonzero constant multiple $c \vec{H}=i_{I}(c \vec{\theta})$ of the canonical coefficients is a solution of the structural equation.

Proof. Suppose $\vec{\theta}$ is a nontrivial homogeneity-0 Frobenius coframe, and $\vec{H}=i_{I} \vec{\theta}$. Then the Levi-Civita connection form $\vec{\Omega}$ is nonzero, and $\vec{F}=i_{E} \vec{\Omega}$ is a nonzero solution of the structural equation by (C1) and (C2). Now (C4) implies $c \vec{H}=\vec{F}$ for some function $c$, but (C2) and (C3) imply $d c=0$, so $c$ is a constant.

Conversely, suppose $c$ is a nonzero constant and that $c \vec{H}$ is a solution of the structural equation. Let $\vec{\theta}$ be the canonical coframe with canonical coefficients $\vec{H}$, and let $\vec{\Omega}$ be the Egoroff connection form with Egoroff coefficients $c \vec{H}$. Since $\vec{F}=i_{E} \vec{\Omega}=c \vec{H}$, this data satisfies conditions (C1)-(C4) of Proposition 4.1, with $m=0$. Therefore $\vec{\theta}$ is a homogeneity-0 Frobenius coframe (and $\vec{\Omega}$ is the Levi-Civita connection form).

It is now an easy matter to prove Proposition 2.2. A simple computation shows that structural equation eq.(4.4) is equivalent to

$$
d f_{1}-\frac{f_{2} f_{3}}{t} d t=0, \quad d f_{2}-\frac{f_{3} f_{1}}{1-t} d t=0, \quad d f_{3}-\frac{f_{1} f_{2}}{t(t-1)} d t=0
$$


where $t$ is the cross-ratio eq.(2.4). Dubrovin [D1; eq.(3.113)] had obtained eq.(4.5) from a Hamiltonian approach to Frobenius manifolds, see also [Hi1]. The same equation, or more precisely its reduction by the $B$-symmetry to an ODE, appears in the work of Tod [T,Hi2] on Riemannian metrics with selfdual curvature in (real) dimension four. Proposition 2.2 is proved by rewriting eq.(4.5) as

$t d f_{1}^{2}=2 f_{1} f_{2} f_{3} d t, \quad(1-t) d f_{2}^{2}=2 f_{1} f_{3} f_{1} d t, \quad t(t-1) d f_{3}^{2}=2 f_{1} f_{2} f_{3} d t=0$

and appealing to Corollary 4.2 to write $c^{2} g_{i i}=f_{i}^{2}$. The trace eq.(2.6) is expressed in terms of $\vec{F}$ by

$$
k=-\frac{c^{2}}{2}\left(g_{11}+g_{22}+g_{33}\right)=-\frac{1}{2}\left(f_{1}^{2}+f_{2}^{2}+f_{3}^{2}\right)=-\frac{1}{2} \vec{F} \cdot \vec{F}
$$

For any solution $\vec{F}$ of the structural equation, it is also clear from the orthogonality of the connection that the trace $k=-\frac{1}{2} \vec{F} \cdot \vec{F}$ is constant.

The trace is related to Dubrovin's $\mu$ by $k=\frac{1}{2} \mu^{2}$, compare [D1; eq.(3.114)]. The three-dimensional Frobenius manifolds associated to the Coxeter groups $A_{3}, B_{3}$, and $H_{3}$ have trace $k=\frac{1}{32}, \frac{2}{25}$, and $\frac{1}{18}$ respectively [D1; App. E]. These values do not appear on the list $k=\frac{1}{2}\left(n+\frac{1}{2}\right)^{2}, n=0,1,2, \ldots$ of Theorem 3.1.

Our final topic is the basic classification theory of three-dimensional Frobenius coframes. An equivalence class $[\vec{\theta}]$ of coframes under the equivalence relation of constant rescaling will be called a "projective coframe". It is evident from the Cartan torsion equation that the Levi-Civita connection form $\vec{\Omega}$ of a coframe $\vec{\theta}$ depends only on the projective coframe $[\vec{\theta}]$, as does $\vec{F}=i_{E} \vec{\Omega}$. This defines a function $\mathcal{S}:[\vec{\theta}] \mapsto \vec{F}$ mapping projective coframes to triplets of functions. It is clear that $\mathcal{S}$ maps Frobenius projective coframes to solutions of the structural equation.

\section{Corollary 4.3.}

(1) The map $\mathcal{S}$ restricts to a bijection from nontrivial homogeneity $m=$ 0 Frobenius projective coframes to nonzero solutions of the structural equation.

(2) For $m \neq 0$, the map $\mathcal{S}$ restricts to a bijection from homogeneity-m Frobenius projective coframes to solutions of the structural equation of trace $k=m^{2} / 8$.

Proof. (1) This follows immediately from Corollary 4.2.

(2) We first show that for nonzero $m, \mathcal{S}$ maps homogeneity- $m$ Frobenius projective coframes to solutions of the structural equation of trace $k=m^{2} / 8$. Suppose $\vec{\theta}$ is a homogeneity- $m$ Frobenius coframe. By (C4), $\vec{H}$ is pointwise an eigenvector with eigenvalue $\lambda=\frac{m}{2}$ of the linear map $M: \vec{H} \mapsto \vec{F} \times \vec{H}$. The identity $\vec{F} \times(\vec{F} \times \vec{H})=(\vec{F} \cdot \vec{H}) \vec{F}-(\vec{F} \cdot \vec{F}) \vec{H}$ gives the characteristic equation 
$M\left(M^{2}-2 k\right)=0$. Now the eigenvalue $\lambda=\frac{m}{2}$ is nonzero, so $\lambda\left(\lambda^{2}-2 k\right)=0$ implies $k=m^{2} / 8$.

We next establish injectivity. Suppose $\vec{\theta}$ and $\vec{\theta}^{\prime}$ are homogeneity- $m$ Frobenius coframes with Levi-Civita connection forms $\vec{\Omega}$ and $\vec{\Omega}^{\prime}$ respectively, and suppose that $\mathcal{S}([\vec{\theta}])=\mathcal{S}\left(\left[\overrightarrow{\theta^{\prime}}\right]\right)$. The Egoroff property $(\mathrm{C} 1)$ of the connection forms then implies $\vec{\Omega}=\vec{\Omega}^{\prime}$. By (C4), the canonical coefficients $\vec{H}=i_{I} \vec{\theta}$ and $\vec{H}^{\prime}=i_{I} \overrightarrow{\theta^{\prime}}$ are pointwise eigenvectors of $M$ with eigenvalue $\frac{m}{2}$. M has three distinct eigenvalues since $k=m^{2} / 8$ is nonzero, so $\vec{H}^{\prime}=c \vec{H}$ for some scalar function $c$, and (C3) implies $d c=0$. Since $\overrightarrow{\theta^{\prime}}=c \vec{\theta}$ for a constant $c$, we have shown that $[\vec{\theta}]=\left[\overrightarrow{\theta^{\prime}}\right]$.

We finally establish surjectivity. Let $\vec{F}$ be a solution of the structural equation of nonzero trace $k=m^{2} / 8$. Let $\vec{\Omega}$ be the Egoroff connection form with Egoroff coefficients $\vec{F}$. Then the Cartan curvature form eq.(4.2) vanishes, see eq.(A.1) below, so the connection is flat. Let $\vec{F}_{p}$ be the value of $\vec{F}$ at at a point $p \in U$, and choose a nonzero $\vec{H}_{p}$ such that $\vec{F}_{p} \times \vec{H}_{p}=\frac{m}{2} \vec{H}_{p}$. Assuming that $U$ is simply connected, parallel transport with the flat connection generates the unique $\vec{H}$ that satisfies condition (C3) and has the value $\vec{H}_{p}$ at $p$. Furthermore $\vec{H}$ satisfies (C4) on $U$, because $\vec{F} \times \vec{H}-\frac{m}{2} \vec{H}$ is covariantly constant by (C2) and (C3), and $\vec{F}_{p} \times \vec{H}_{p}-\frac{m}{2} \vec{H}_{p}=0$. This data satisfies conditions (C1)-(C4) of Proposition 4.1 , so the canonical coframe $\vec{\theta}$ with canonical coefficients $\vec{H}$ is a homogeneity- $m$ Frobenius coframe such that $\mathcal{S}([\vec{\theta}])=\vec{F}$.

To summarize, a trace- $k$ solution $\vec{F}$ of the structural equation generates a homogeneity- $m$ Frobenius coframe for each distinct root $m$ of $m\left(m^{2}-8 k\right)=0$. The explicit construction of the homogeneity- 0 Frobenius coframe from $\vec{F}$ is trivial by Corollary 4.2. The explicit construction of the nonzero homogeneity Frobenius coframes is somewhat more complicated, see Hitchin [Hi].

\section{Appendix: Proof of Proposition 4.1}

To prove Proposition 4.1, we need to establish that the conditions (C1)-(C4) together with the Cartan torsion equation are equivalent to the conditions (M1)(M3).

Lemma A.1. If (C1)-(C4) hold, then $d \vec{\theta}-\vec{\Omega} \times \vec{\theta}=0$.

Proof. The proof only requires conditions (C1) and (C3). The first component of the torsion of a canonical coframe is

$$
d \theta_{1}-\Omega_{2} \theta_{3}+\Omega_{3} \theta_{2}=d\left(h_{1} d x_{1}\right)-\Omega_{2} \wedge\left(h_{3} d x_{3}\right)+\Omega_{3} \wedge\left(h_{2} d x_{2}\right) .
$$

The Egoroff condition (C1) is equivalent to

$$
\Omega_{1} \wedge\left(d x_{2}-d x_{3}\right)=0, \quad \Omega_{2} \wedge\left(d x_{3}-d x_{1}\right)=0, \quad \Omega_{3} \wedge\left(d x_{1}-d x_{2}\right)=0,
$$


and we have

$$
d \theta_{1}-\Omega_{2} \theta_{3}+\Omega_{3} \theta_{2}=\left(d h_{1}-\Omega_{2} h_{3}+\Omega_{3} h_{2}\right) \wedge d x_{1},
$$

which vanishes by (C3). The other components of the torsion vanish analogously.

We may therefore assume $d \vec{\theta}-\vec{\Omega} \times \vec{\theta}=0$, and establish $((\mathrm{C} 1)-(\mathrm{C} 4)) \Leftrightarrow((\mathrm{M} 1)-$ (M3)) under this assumption. We will break this up into a number of separate steps:

(1) $(\mathrm{C} 3) \Leftrightarrow(\mathrm{M} 3)$

(2) $((\mathrm{C} 3)$ and $(\mathrm{C} 4)) \Leftrightarrow((\mathrm{M} 3)$ and $(\mathrm{M} 2))$

(3) $((\mathrm{C} 1)$ and $(\mathrm{C} 2)) \Rightarrow(\mathrm{M} 1)$

(4) $(\mathrm{C} 2) \Leftarrow((\mathrm{M} 1)$ and $(\mathrm{M} 2))$

(5) $(\mathrm{C} 3) \Rightarrow(\mathrm{C} 1)$

Proof of $(\mathrm{C} 3) \Leftrightarrow(\mathrm{M} 3) . I=h_{1} e_{1}+h_{2} e_{2}+h_{3} e_{3}$, so the covariant constancy of $I$ is equivalent to $\vec{\nabla} \vec{H}=d \vec{H}-\vec{\Omega} \times \vec{H}=0$.

Proof of $((\mathrm{C} 3)$ and $(\mathrm{C} 4)) \Leftrightarrow\left((\mathrm{M} 3)\right.$ and (M2)). (M2) is equivalent to $\mathcal{L}_{E} \vec{H}=\frac{m}{2} \vec{H}$. If (C3) or equivalently (M3) holds, then

$$
0=i_{E}(d \vec{H}-\vec{\Omega} \times \vec{H})=\mathcal{L}_{E} \vec{H}-\vec{F} \times \vec{H},
$$

and then (M2) is equivalent to (C4).

Proof of $((\mathrm{C} 1)$ and $(\mathrm{C} 2)) \Rightarrow(\mathrm{M} 1)$. Substitute $\Omega_{i}$ from eq.(4.3) into the first component of the Cartan curvature equation eq.(4.2) and use eq.(4.4);

$$
\begin{aligned}
R_{1} & =d \Omega_{1}-\frac{1}{2}\left(\Omega_{2} \wedge \Omega_{3}-\Omega_{3} \wedge \Omega_{2}\right) \\
& =d f_{1} \wedge\left(\frac{d x_{2}-d x_{3}}{x_{2}-x_{3}}\right)-f_{2} f_{3}\left(\frac{d x_{3}-d x_{1}}{x_{3}-x_{1}}\right) \wedge\left(\frac{d x_{1}-d x_{2}}{x_{1}-x_{2}}\right) \\
& =-f_{2} f_{3} \frac{i_{E}\left(\left(d x_{2}-d x_{3}\right) \wedge\left(d x_{3}-d x_{1}\right) \wedge\left(d x_{1}-d x_{2}\right)\right)}{\left(x_{2}-x_{3}\right)\left(x_{3}-x_{1}\right)\left(x_{1}-x_{2}\right)}
\end{aligned}
$$

which vanishes because $\left(d x_{2}-d x_{3}\right) \wedge\left(d x_{3}-d x_{1}\right) \wedge\left(d x_{1}-d x_{2}\right)=0$. The other components of the curvature form $\vec{R}$ vanish similarly.

Recall that a vector field $B$ is said to be a "conformal Killing vector" for the Riemannian metric $g$ if $\mathcal{L}_{B} g=r g$ for some constant $r$. The following standard lemma holds in any dimension.

Lemma A.2. If $B$ is a conformal Killing vector for a flat Riemannian metric $g$, then the tangent bundle endomorphism $\nabla B$ is covariantly constant.

Proof. A flat Riemannian metric locally admits "flat coordinates" $\left\{t_{i}\right\}$ such that $\left\{\epsilon_{i}=\frac{\partial}{\partial t_{i}}\right\}$ is an orthonormal frame. The basis vector fields are covariantly 
constant, $\nabla \epsilon_{i}=0$, and the covariant derivatives along the basis vectors commute, $\nabla_{\epsilon_{i}} \nabla_{\epsilon_{j}}=\nabla_{\epsilon_{j}} \nabla_{\epsilon_{i}}$. The metric equals $g=\sum_{j} d t_{j} \otimes d t_{j}$, where $\left\{d t_{i}\right\}$ is the dual coframe.

Writing $B=\sum_{k} b_{k} \epsilon_{k}$, we have $\mathcal{L}_{B} d t_{j}=d\left(\mathcal{L}_{B} t_{j}\right)=d b_{j}=\sum_{k}\left(\nabla_{\epsilon_{k}} b_{j}\right) d t_{k}$, and

$$
\mathcal{L}_{B} g=\sum_{j} \mathcal{L}_{B}\left(d t_{j} \otimes d t_{j}\right)=\sum_{j, k}\left(\nabla_{\epsilon_{j}} b_{k}+\nabla_{\epsilon_{k}} b_{j}\right) d t_{j} \otimes d t_{k}
$$

Since $r$ is constant and $g$ is covariantly constant, $\mathcal{L}_{B} g=r g$ implies

$$
0=\nabla_{\epsilon_{i}}\left(\mathcal{L}_{B} g\right)=\sum_{j, k}\left(\nabla_{\epsilon_{i}} \nabla_{\epsilon_{j}} b_{k}+\nabla_{\epsilon_{k}} \nabla_{\epsilon_{i}} b_{j}\right) d t_{j} \otimes d t_{k}
$$

After several permutations of the indices,

$$
\nabla_{\epsilon_{i}} \nabla_{\epsilon_{j}} b_{k}=-\nabla_{\epsilon_{k}} \nabla_{\epsilon_{i}} b_{j}=\nabla_{\epsilon_{j}} \nabla_{\epsilon_{k}} b_{i}=-\nabla_{\epsilon_{i}} \nabla_{\epsilon_{j}} b_{k}
$$

which implies $\nabla_{\epsilon_{i}} \nabla_{\epsilon_{j}} b_{k}=0$ for any $i, j, k$. We conclude that $\nabla B$ is covariantly constant, as

$$
\nabla_{\epsilon_{i}}(\nabla B)=\sum_{j, k}\left(\nabla_{\epsilon_{i}} \nabla_{\epsilon_{j}} b_{k}\right) d t_{j}=0
$$

Proof of $(C 2) \Leftarrow((M 1)$ and $(M 2))$. Assume (M1) and (M2) hold. Since $\mathcal{L}_{E} d x_{i}=$ $d x_{i}$, (M2) implies $\mathcal{L}_{E} g=(m+2) g$. From (M1) and Lemma A.2 we conclude that $\nabla E$ is covariantly constant. We will now show that $\nabla E$ is covariantly constant only if (C2) holds.

We compute $\nabla_{Z}(\nabla E)$ for an arbitrary vector field $Z$. From the Leibniz property

$$
\nabla_{Z}\langle\nabla E, Y\rangle=\left\langle\nabla_{Z}(\nabla E), Y\right\rangle+\left\langle\nabla E, \nabla_{Z} Y\right\rangle
$$

conclude that

$$
\left\langle\nabla_{Z}(\nabla E), Y\right\rangle=\nabla_{Z} \nabla_{Y} E-\nabla_{\left(\nabla_{Z} Y\right)} E
$$

We compute $\nabla_{V} E$ for an arbitrary vector field $V$. The vanishing torsion of the Levi-Civita connection gives

$$
\nabla_{V} E=\nabla_{E} V-\mathcal{L}_{E} V
$$

Now $e_{i}=h_{i}^{-1} \partial_{i}$, so using the Leibniz property of the Lie derivative and the homogeneity property $\mathcal{L}_{E} h_{i}^{-1}=-\frac{m}{2} h_{i}^{-1}$, we have $\mathcal{L}_{E} e_{i}=-\beta e_{i}$, where $\beta=$ $\left(\frac{m}{2}+1\right)$, so

$$
\begin{aligned}
\mathcal{L}_{E} V & =\mathcal{L}_{E}\left(V_{1} e_{1}+V_{2} e_{2}+V_{3} e_{3}\right) \\
& =\left(\mathcal{L}_{E} V_{1}-\beta V_{1}\right) e_{1}+\left(\mathcal{L}_{E} V_{2}-\beta V_{2}\right) e_{2}+\left(\mathcal{L}_{E} V_{3}-\beta V_{3}\right) e_{3} .
\end{aligned}
$$


Now eq.(A.3) written in in terms of components is

$$
\begin{aligned}
\vec{\nabla}_{V} \vec{E} & =\left(\mathcal{L}_{E} \vec{V}-\left(i_{E} \vec{\Omega}\right) \times \vec{V}\right)-\left(\mathcal{L}_{E} \vec{V}-\beta \vec{V}\right) \\
& =-\vec{F} \times \vec{V}+\beta \vec{V}
\end{aligned}
$$

Setting $V=Y$ in eq.(A.4) and applying $\vec{\nabla}_{Z}$ gives

$$
\vec{\nabla}_{Z} \vec{\nabla}_{Y} \vec{E}=-\left(\vec{\nabla}_{Z} \vec{F}\right) \times \vec{Y}-\vec{F} \times\left(\vec{\nabla}_{Z} \vec{Y}\right)+\beta \vec{\nabla}_{Z} \vec{Y}
$$

while setting $V=\nabla_{Z} Y$ in eq.(A.4) gives

$$
\vec{\nabla}_{\left(\nabla_{Z} Y\right)} \vec{E}=-\vec{F} \times\left(\vec{\nabla}_{Z} \vec{Y}\right)+\beta \vec{\nabla}_{Z} \vec{Y}
$$

and from eq.(A.2) we conclude that $\left\langle\nabla_{Z}(\nabla E), Y\right\rangle=0$ if and only if $\left(\vec{\nabla}_{Z} \vec{F}\right) \times$ $\vec{Y}=0$. Since $Y$ and $Z$ are arbitrary, $\nabla E$ is covariantly constant if and only if $0=\vec{\nabla} \vec{F}=d \vec{F}-\vec{\Omega} \times \vec{F}$. This is just the condition (C2).

The following lemma will complete the proof of Proposition 4.1, and also establish the equivalence of our definition of the Egoroff condition, eq.(4.3), with the usual definition, (C1a) below.

Lemma A.3. Let $\vec{\theta}$ be a canonical coframe with canonical coefficients $\vec{H}=i_{I} \vec{\theta}$, and let $\vec{\Omega}$ be its Levi-Civita connection form. Then the following are equivalent.

(C1) $\vec{\Omega}$ is an Egoroff connection form.

(C1a) The one-form $\vec{H} \cdot \vec{\theta}=g_{11} d x_{1}+g_{22} d x_{2}+g_{33} d x_{3}$ is closed.

(C1b) $(d \vec{H}-\vec{\Omega} \times \vec{H}) \cdot \vec{\theta}=0$.

(C1c) $i_{I} \vec{\Omega}=0$.

Proof. The equivalence $(\mathrm{C} 1 \mathrm{a}) \Leftrightarrow(\mathrm{C} 1 \mathrm{~b})$ is immediate from the derivation property of $d$ and the triple-product identity:

$$
d(\vec{H} \cdot \vec{\theta})=(d \vec{H}) \cdot \vec{\theta}+\vec{H} \cdot(d \vec{\theta})=(d \vec{H}) \cdot \vec{\theta}+\vec{H} \cdot(\vec{\Omega} \times \vec{\theta})=(d \vec{H}-\vec{\Omega} \times \vec{H}) \cdot \vec{\theta} .
$$

To prove $(\mathrm{C} 1 \mathrm{~b}) \Leftrightarrow(\mathrm{C} 1 \mathrm{c})$, start with

$$
\begin{aligned}
d \vec{H}-\vec{\Omega} \times \vec{H} & =d i_{I} \vec{\theta}-\vec{\Omega} \times\left(i_{I} \vec{\theta}\right) \\
& =\left(\mathcal{L}_{I} \vec{\theta}-i_{I} d \vec{\theta}\right)+i_{I}(\vec{\Omega} \times \vec{\theta})-\left(i_{I} \vec{\Omega}\right) \times \vec{\theta} \\
& =\mathcal{L}_{I} \vec{\theta}-\left(i_{I} \vec{\Omega}\right) \times \vec{\theta}-i_{I}(d \vec{\theta}-\vec{\Omega} \times \vec{\theta}) \\
& =\mathcal{L}_{I} \vec{\theta}-\left(i_{I} \vec{\Omega}\right) \times \vec{\theta},
\end{aligned}
$$

which implies

$$
(d \vec{H}-\vec{\Omega} \times \vec{H}) \cdot \vec{\theta}=\left(\mathcal{L}_{I} \vec{\theta}-\left(i_{I} \vec{\Omega}\right) \times \vec{\theta}\right) \cdot \vec{\theta}=\left(\mathcal{L}_{I} \vec{\theta}\right) \cdot \vec{\theta}-\left(i_{I} \vec{\Omega}\right) \cdot(\vec{\theta} \times \vec{\theta}) .
$$


Now

$\left(\mathcal{L}_{I} \vec{\theta}\right) \cdot \vec{\theta}=\left(\mathcal{L}_{I} h_{1}\right) h_{1} d x_{1} \wedge d x_{1}+\left(\mathcal{L}_{I} h_{2}\right) h_{2} d x_{2} \wedge d x_{2}+\left(\mathcal{L}_{I} h_{2}\right) h_{2} d x_{2} \wedge d x_{2}=0$

and

$$
\left(i_{I} \vec{\Omega}\right) \cdot(\vec{\theta} \times \vec{\theta})=2\left(i_{I} \Omega_{1}\right) \theta_{2} \wedge \theta_{3}+2\left(i_{I} \Omega_{2}\right) \theta_{3} \wedge \theta_{1}+2\left(i_{I} \Omega_{3}\right) \theta_{1} \wedge \theta_{2},
$$

so $(d \vec{H}-\vec{\Omega} \times \vec{H}) \cdot \vec{\theta}$ vanishes if and only if each component of $i_{I} \vec{\Omega}$ vanishes.

Finally we prove (C1c) $\Leftrightarrow(\mathrm{C} 1)$. Any coframe $\vec{\theta}$ and dual frame $\vec{e}$ tautologically satisfy $i_{e_{i}} \theta_{j}=\delta_{i j}$. A canonical coframe has the additional property $i_{e_{2}} i_{e_{3}} d \theta_{1}=$ 0 , which follows from $d \theta_{1}=h_{1}^{-1} d h_{1} \wedge \theta_{1}$. The first component of the Cartan torsion equation gives

$$
0=i_{e_{2}} i_{e_{3}}\left(d \theta_{1}-\Omega_{2} \wedge \theta_{3}+\Omega_{3} \wedge \theta_{2}\right)=i_{e_{2}} \Omega_{2}+i_{e_{3}} \Omega_{3}
$$

which together with the other components $i_{e_{3}} \Omega_{3}+i_{e_{1}} \Omega_{1}=0$ and $i_{e_{1}} \Omega_{1}+$ $i_{e_{2}} \Omega_{2}=0$ implies $i_{e_{i}} \Omega_{i}=0$, or equivalently $i_{\partial_{i}} \Omega_{i}=0$. So the connection form of a canonical coframe satisfies

$$
\vec{\Omega}=\left(\begin{array}{c}
i_{\partial_{2}} \Omega_{1} d x_{2}+i_{\partial_{3}} \Omega_{1} d x_{3} \\
i_{\partial_{3}} \Omega_{2} d x_{3}+i_{\partial_{1}} \Omega_{2} d x_{1} \\
i_{\partial_{1}} \Omega_{3} d x_{1}+i_{\partial_{2}} \Omega_{3} d x_{2}
\end{array}\right), \quad i_{I} \vec{\Omega}=\left(\begin{array}{c}
i_{\partial_{2}} \Omega_{1}+i_{\partial_{3}} \Omega_{1} \\
i_{\partial_{3}} \Omega_{2}+i_{\partial_{1}} \Omega_{2} \\
i_{\partial_{1}} \Omega_{3}+i_{\partial_{2}} \Omega_{3}
\end{array}\right)
$$

Now $(\mathrm{C} 1 \mathrm{c})$ is equivalent to

$$
i_{\partial_{3}} \Omega_{1}=-i_{\partial_{2}} \Omega_{1}, \quad i_{\partial_{1}} \Omega_{2}=-i_{\partial_{3}} \Omega_{2}, \quad i_{\partial_{2}} \Omega_{3}=-i_{\partial_{1}} \Omega_{3},
$$

which is equivalent to (C1), compare with eq.(4.3):

$$
\vec{\Omega}=\left(\begin{array}{c}
f_{1}\left(x_{2}-x_{3}\right)^{-1}\left(d x_{2}-d x_{3}\right) \\
f_{2}\left(x_{3}-x_{1}\right)^{-1}\left(d x_{3}-d x_{1}\right) \\
f_{3}\left(x_{1}-x_{2}\right)^{-1}\left(d x_{1}-d x_{2}\right)
\end{array}\right), \quad \vec{F}=\left(\begin{array}{c}
f_{1} \\
f_{2} \\
f_{3}
\end{array}\right)=\left(\begin{array}{c}
\left(x_{2}-x_{3}\right) i_{\partial_{2}} \Omega_{1} \\
\left(x_{3}-x_{1}\right) i_{\partial_{3}} \Omega_{2} \\
\left(x_{1}-x_{2}\right) i_{\partial_{1}} \Omega_{3}
\end{array}\right)
$$

Proof of $(\mathrm{C} 3) \Rightarrow(\mathrm{C} 1)$. Obviously $(\mathrm{C} 3) \Rightarrow(\mathrm{C} 1 \mathrm{~b})$, and $(\mathrm{C} 1 \mathrm{~b}) \Leftrightarrow(\mathrm{C} 1)$ by Lemma A.3.

This completes the proof of Proposition 4.1.

\section{References}

[ADHM] M. F. Atiyah, V. G. Drinfeld, N. J. Hitchin, and Yu. I. Manin, Construction of Instantons, Phys. Lett. 65A (1978), 185.

[At] M. F. Atiyah, Geometry of Yang-Mills Fields, Lezioni Fermiane, Accademia Nazionale dei Lince \& Scuola Normale Superiore, Pisa, 1979. 
[Au] M. Audin, An introduction to Frobenius manifolds, moduli spaces of stable maps and quantum cohomology, Preprint.

[BS] G. Bor and J. Segert, Symmetric instantons and the ADHM construction, Comm. Math. Phys 183 (1997), 183-203.

[CDD] Y. Choquet-Bruhat, C. DeWitt-Morette, and M. Dillard-Bleick, Analysis, Manifolds and Physics, revised edition, North-Holland, Amsterdam, 1982.

[D1] B. Dubrovin, Geometry of $2 D$ topological field theories, Lecture Notes in Mathematics, vol. 1620, Springer-Verlag, 1996, pp. 120-348.

[D2] - Painlevé transcendents and two-dimensional topological field theory, Preprint, math.AG/9803017 (1998).

[F] R. Fuchs, Über lineare homogene Differentialgleichungen zweiter Ordnung mit drei im Endlichen gelegenen wesentlich singulären Stellen, Math. Ann. 63 (1907), 301321.

[G] A. B. Givental, Equivariant Gromov-Witten invariants, Internat. Math. Res. Notices 13 (1996), 613-663.

[Hi1] N. J. Hitchin, Frobenius manifolds (Notes by David Calderbank), Preprint (1996).

[Hi2] - Twistor spaces, Einstein metrics and isomonodromic deformations, J. Diff. Geom. 42 (1995), 30-112.

[Hi3] $\quad$ Poncelet polygons and the Painlevé equations, Geometry and Analysis, Tata Institute of Fundamental Research, Bombay, pp. 151-185.

[JM] M. Jimbo and T. Miwa, Monodromy preserving deformations of linear ordinary differential equations with rational coefficients. II, Physica 2D (1981), 407-448.

[Ml] B. Malgrange, Sur les deformations isomonodromiques I. Singularités régulières, Mathématique et Physique, Séminaire de l'Ecole Normale Supérieure 1979-1982, Progress in Mathematics, vol. 37, Birkhäuser, 1983, pp. 401-426.

[Mn1] Yu. I. Manin, Frobenius manifolds, quantum cohomology, and moduli spaces, Preprint MPI 96-113.

[Mn2] Sixth Painlevé equation, universal elliptic curve, and mirror of $P^{2}$, Preprint math.AG/9605010 (1996).

[Mn3] - Three constructions of Frobenius manifolds: a comparative study, Preprint, math.AG/9801006 (1998).

[Ro] J. Rotman, Galois Theory, Springer-Verlag, New York, 1990.

[RuT] Y. Ruan and G. Tian, A mathematical theory of quantum cohomology, J. Diff. Geom 42 (1995), 269-367.

[Sa] C. Sabbah, Frobenius manifolds: isomonodromic deformations and infinitesimal period mappings, Preprint.

[Se] J. Segert, Painlevé solutions from equivariant holomorphic bundles, Preprint (1996).

[T] K. P. Tod, Self-dual Einstein metrics from the Painlevé VI equation, Phys. Lett A 190 (1994), 221-224.

Department of Mathematics, University of Missouri, Columbia, MO 65211

E-mail address: jan@math.missouri.edu 